\title{
Do Changes in Post-acute Care Use at Hospitals Participating in an Accountable Care Organization Spillover to All Medicare Beneficiaries?
}

\author{
Amol S. Navathe, MD, PhD 1,2,3,4, Alexander M. Bain, B. $A^{2}$, and Rachel M. Werner, MD, PhD ${ }^{1,2,3}$ \\ 'Corporal Michael J. Cresencz VA Medical Center, Philadelphia, PA, USA; ${ }^{2}$ Perelman School of Medicine, University of Pennsylvania, Philadelphia, \\ PA, USA; ${ }^{3}$ Leonard Davis Institute of Health Economics, The Wharton School, University of Pennsylvania, Philadelphia, PA, USA; ${ }^{4}$ Division of Health \\ Policy, University of Pennsylvania, Philadelphia, PA, USA.
}

BACKGROUND: While early evidence suggests that Medicare accountable care organizations (ACOs) may reduce post-acute care (PAC) utilization for attributed beneficiaries, whether these effects spill over to all beneficiaries admitted to hospitals participating in ACOs stray is unknown.

OBJECTIVE: The objective of this study was to evaluate whether changes in PAC use and Medicare spending spill over to all beneficiaries admitted to hospitals participating in the Medicare Shared Savings Program (MSSP).

DESIGN: Observational study using a difference-indifferences design comparing changes in PAC utilization and spending among beneficiaries admitted to ACOparticipating hospitals before and after the start of the ACO contracts, compared to those admitted to non-ACO hospitals.

SETTING: A total of 233 hospitals participate in MSSP ACOs and 3103 non-ACO hospitals.

PARTICIPANTS: A national sample of 11,683,573 Medicare beneficiaries experiencing 26,503,086 hospital admissions from 2010 to 2013.

EXPOSURE: Admission to a hospital participating in an MSSP ACO.

MAIN MEASURES: The probability of discharge and Medicare payments to inpatient rehabilitation facilities (IRF), skilled nursing facilities (SNF), and home health agencies (HHA).

KEY RESULTS: For beneficiaries admitted to hospitals that joined an ACO, the likelihood of being discharged to PAC did not change after the hospital joined the ACO compared with non-ACO hospitals over the same period (differential change in probability of discharge to any PAC was $0.000(P=0.89)$, SNF was $0.000(P=0.73)$, IRF was $0.000(P=0.96)$, and HHA was $0.001(P=0.57))$. Payments reduced significantly for $\mathrm{PAC}$ overall $(-\$ 130.41, P=0.03)$, but not for any individual PAC type alone. These results were consistent in samples that were conditional on discharge to any PAC, across conditions with high PAC use nationally, and among ACO-participating hospitals that also had a PAC participant.

Electronic supplementary material The online version of this article (https://doi.org/10.1007/s11606-018-4368-z) contains supplementary material, which is available to authorized users.

Received July 21, 2017

Revised October 27, 2017

Accepted January 31, 2018

Published online March 8, 2018
CONCLUSIONS: Hospital participation in an ACO did not result in spillovers in PAC utilization or payments to all beneficiaries, even when considering high PAC-use conditions and ACO hospitals that also have an ACOparticipating PAC.

KEY WORDS: accountable care organization; post-acute care; skilled nursing facility; Medicare; health policy.

J Gen Intern Med 33(6):831-8

DOI: $10.1007 / \mathrm{s} 11606-018-4368-\mathrm{z}$

() Society of General Internal Medicine 2018

\section{INTRODUCTION}

Medicare payments for post-acute care (PAC), totalling \$60 billion in 2015, ${ }^{1}$ have grown faster than most other categories of spending, and a 2013 Institute of Medicine report indicated that PAC utilization is the largest driver of variation in Medicare spending. ${ }^{2,3}$ Value-based health care payment policies may help reduce the unwarranted variation in PAC spending, quality, and utilization by reducing unnecessary utilization. One of the Medicare's largest experiments with value-based payment is its most prevalent accountable care organization (ACO) model, the Medicare Shared Savings Program (MSSP). Prior work has shown that this ACO model was associated with reductions in overall Medicare payments and stable-to-improved quality for beneficiaries attributed to its ACOs. ${ }^{4,5}$ Early evidence also suggests that these ACOs decrease institutional PAC utilization, including skilled nursing facilities (SNFs), inpatient rehabilitation facilities (IRFs), and long-term care hospitals, as well as reduced spending on SNFs for attributed beneficiaries. ${ }^{6}$

While over 9 million Medicare beneficiaries are directly affected by ACOs through attribution, this represents a small fraction of the 57.5 million beneficiaries nationwide. ${ }^{7,8}$ One goal of ACO policies is to encourage system-wide practice transformation to improve the value of care for all patients, not just those attributed to the ACO, though the financial incentives through MSSP may be small. However, whether all beneficiaries admitted to hospitals participating in Medicare ACOs experience these beneficial spillovers is unknown, but is of vital importance in evaluating the overall impact of ACOs. 
The interface between hospitals and PAC providers may be a particularly important transition point in the quest for value under ACOs. On one hand, PAC providers can provide more intensive care through the transition out of the hospital than is possible without formal PAC. On the other hand, without financial accountability, hospitals may not have the incentive to match patients with the best post-discharge location of care, including whether or not to use PAC and the most appropriate level of PAC.

Thus, our objective was to evaluate whether Medicare beneficiaries discharged from a hospital participating in the MSSP ACO program had different patterns of postacute care utilization compared to Medicare beneficiaries admitted to hospitals not participating in an MSSP ACO, regardless of whether the beneficiary was attributed to an MSSP ACO or not. While prior literature has documented reductions in PAC utilization from MSSP-attributed beneficiaries, ${ }^{6}$ our approach tests whether ACO hospitals are generating systematic changes in PAC utilization that spill over to the broader Medicare population regardless of ACO attribution status.

\section{METHODS}

\section{Data}

We used patient-level data for all Medicare hospital discharges and PAC use from Medicare claims nationally between 2010 and 2013 (from the 100\% MedPAR file and Home Health Agency (HHA) claims file). These claims data were supplemented with the Medicare Beneficiary Summary File, containing information on beneficiary enrollment and demographics (age, sex, race, or ethnic group). ${ }^{9}, 10$

Provider-level data were obtained from two sources. First, to determine hospital and PAC provider participation in the MSSP, we obtained publicly available lists from the Centers for Medicare and Medicaid Services (CMS) of participants that joined MSSP ACOs from April 2012 through January 2013. We then categorized whether or not each ACO included a hospital (designated "ACO hospitals") and, for ACO hospitals, the date the ACO joined the MSSP. ACOs joined the MSSP in three waves during our study period: those that joined on (1) April 1, 2012, (2) July 1, 2012, and (3) January 1, 2013. For each ACO hospital, we also identified whether the ACO included a SNF, IRF, and/or HHA. In cases where it was unclear how to categorize the ACO participant (e.g., if a hospital was included as a participant, and that hospital had an IRF located within it but the IRF was not listed separately as a participant), we contacted the ACO directly by phone and email to clarify participation. Because only eight ACO hospitals co-participated with a SNF alone, this category was excluded.

The second source of provider data was the 2013 Provider of Service File, from which we drew hospital and PAC facility characteristics used for descriptive purposes such as the teaching status (resident-to-bed ratio) and size (e.g., number of beds or number of staff).

\section{Study Population}

We included all acute care hospitals and Medicare-certified PAC providers that admitted Medicare beneficiaries in each year between 2010 and 2013. This resulted in 3336 hospitals, 15,276 SNFs, 1216 IRFs, and 9651 HHAs. The 3336 hospitals comprised 233 ACO hospitals and 3103 non-ACO hospitals during our study years. Within each hospital, we created four cohorts of Medicare fee-for-service (FFS) beneficiaries: those discharged to SNF, IRF, or HHA within 2 days of hospital discharge and those with another discharge disposition such as home without formal services or patients transferred to another acute hospital.

We excluded beneficiaries who died in the hospital (as they could not be discharged to PAC), younger than the age of 65 (to create a more homogenous population), or enrolled in Medicare Advantage in the 1 year prior to hospital discharge (the period during which we measure patient comorbidities) to ensure that claims are completely identified. We further excluded beneficiaries discharged to hospice, discharged from the hospital against medical advice, or admitted for rehabilitation care or fitting of prostheses and adjustment devices as PAC utilization would not be as relevant for this group.

We also separately examined beneficiaries with conditions that frequently lead to use of PAC by selecting conditions that are among the 10 most frequent reasons for hospitalization admitted to SNF, IRF, and HHA. Patients were grouped by their Medical Severity Diagnosis-Related Group (MS-DRG) including major joint replacement of lower extremity ("major joint replacement") (MS-DRG 469 and 470), hip and femur fracture surgical repair ("hip fracture surgery") (MS-DRG 480-482), coronary artery bypass graft surgery (CABG) (MS-DRG 231-236), congestive heart failure (CHF) (MSDRG 291-293), chronic obstructive pulmonary disease (COPD) (MS-DRG 190-192, 202, and 203), and acute myocardial infarction (AMI) (MS-DRG 280-282). Our final subgroup was patients with a high severity of illness, defined as having an Elixhauser comorbidity score in the top $20 \%$ within the sample. ${ }^{11}$

\section{Post-Acute Care Utilization and Payments}

We used five primary outcome variables: probability of discharge to any PAC (including SNF, IRF, and HHA), probability of discharge to IRF, probability of discharge to SNF, probability of discharge to HHA, and probability of discharge to "institutional PAC" that comprised IRF and SNF discharges. We also evaluated the probability of discharge to each PAC type (SNF, IRF, HHA, and IRF/SNF combined) conditional on discharge to any PAC to evaluate whether PAC utilization shifted among beneficiaries for whom the decision to discharge to PAC was already made. PAC payments were measured as Medicare payments over 60 days post-discharge for each of SNF, IRF, and HHA alone and all PAC combined. Other secondary outcomes included length of stay in SNF and IRF (in days) and number of visits for HHA. 


\section{Market Characteristics}

We used several time-varying market-level variables to control for factors that could be associated with ACO participation and changes in PAC. For each year, we measured the share of admissions within an HRR that go to an ACO hospital to control for ACO penetration in a market, each hospital's Herfindahl-Hirschman Index (HHI) based on hospital discharges to account for market concentration, the number of each PAC type (SNF, IRF, HHA) to adjust for PAC capacity in the market, and aggregate PAC HHI based on PAC beds for SNF and IRF and staffing (full time equivalents) for HHA to account for PAC market concentration.

\section{Risk Adjustment}

We controlled for patient demographics (age, sex, race), 31 Elixhauser comorbidities, dual eligibility for Medicare and Medicaid, urban or rural beneficiary ZIP code of residence, DRG fixed effects (i.e., a dummy variable for each DRG), hospital fixed effects, time varying HRR/market characteristics, and a time fixed effect for each 6-month period during our study. Hospital fixed effects controlled for all unobservable, time-invariant hospital characteristics. Time fixed effects controlled for national secular trends.

\section{Statistical Analysis}

We compared patient-level variables pre- and post-ACO participation for patients at ACO-hospitals and prior to and after January 1, 2013 (the entry date of the third wave of ACOs in our sample) for the non-ACO hospitals. We also examined the hospital and market characteristics for $\mathrm{ACO}$ versus non- $\mathrm{ACO}$ hospitals. Unadjusted trends in the proportion of discharges to PAC overall, IRF, SNF, HHA, and other dispositions were evaluated using line graphs for each wave of ACO hospitals and non-ACO hospitals.

For the primary analysis, we implemented a difference-indifferences design using a linear probability model to compare changes in the probability of PAC utilization among patients receiving care at 233 hospitals participating in an $\mathrm{ACO}$ before and after their ACO participation began to changes in outcomes among patients receiving care at 3103 hospitals not participating in an ACO (comparison group) over the same time period. Importantly, this approach examines the pre-post changes among beneficiaries admitted to the same hospital, allowing each hospital to serve as a control for itself, and for the same condition (MS-DRG), since the indication for PAC may vary widely by the clinical reason for admission. We also examined the effect of ACO participation on length of stay and Medicare payment for PAC.

We repeated this analysis for specific patient subgroups including patients in the top $20 \%$ of illness severity ${ }^{11}$ and with specific medical and surgical conditions that represent a large share of PAC admissions nationally: major joint replacement, hip fracture surgery, CABG, CHF,
COPD, and AMI. Next, we repeated the difference-indifferences analysis conditional on discharge to PAC to evaluate whether composition of PAC type changed among patients using PAC. Finally, we examined the effect of ACO participation when hospitals and PAC providers co-participate in the same ACO-because ACOs with both hospitals and PAC providers may respond to ACO participation differently than ACO hospitals without a co-participating PAC provider.

We conducted several additional analyses to evaluate the robustness of our design and results. Because the difference-in-differences specification assumes that there will be parallel trends in measured outcomes in the absence of the ACO intervention, we tested whether trends in PAC utilization between ACO and non-ACO hospitals were similar during the pre-ACO period (2010-2011). We test this graphically and by testing for differences in trends with regressions for each outcome on binary variables for 6-month time periods, a binary variable indicating 1 for eventual ACO participation, and their interaction. We also included the control variables used in the main analysis. Since ACOs may affect the number of patients hospitalized in a market, we tested for differences in the number of admissions based on ACO participation at the hospital and market levels and examined whether patterns of PAC utilization changed differentially in ACO markets versus markets without ACOs.

All analyses used clustered standard errors to account for multiple patients per hospital and used the Huber-White correction for heteroscedasticity. Analyses were conducted using STATA (version 14.2, College Station, TX) and SAS (version 9.4, Cary, NC). All tests of significance were two-tailed at an alpha of 0.05 . The University of Pennsylvania Institutional Review Board approved the study.

\section{RESULTS}

\section{Sample Characteristics}

Between 2010 and 2013, 11,683,573 unique Medicare beneficiaries experienced 26,503,086 discharges across 3336 acute care hospitals, of which 2,933,534 discharges $(11.1 \%)$ occurred at the 233 ACO hospitals (Table 1). In the pre-ACO period (or prior to January 1, 2013 for the non-ACO hospitals), beneficiaries going to ACO versus non-ACO hospitals were similar in most characteristics, including age, sex, percent white, and Elixhauser comorbidities. There were notable differences in terms of percent black (8.4 versus $9.7 \%$, respectively), dual eligible (21.4 versus $23.5 \%$ ), and residence in an urban location (94.5 versus $91.6 \%$ ). The distribution of PAC use was similar across the two hospital groups, though non-ACO hospitals had slightly lower use of PAC overall in the early period compared to ACO hospitals. Despite these differences, there were no notable differences in the 
Table 1 Sample Characteristics of Medicare Beneficiaries Based on Admission to ACO and Non-ACO Hospitals from 2010 to 2013

\begin{tabular}{|c|c|c|c|c|}
\hline & $\begin{array}{l}\text { Admission to pre- } \\
\text { ACO }\end{array}$ & $\begin{array}{l}\text { Admission to post- } \\
\text { ACO }\end{array}$ & $\begin{array}{l}\text { Non-ACO pre } 01 / 01 / \\
2013\end{array}$ & $\begin{array}{l}\text { Non-ACO post } 01 / 01 \\
2013\end{array}$ \\
\hline Unique benificiaries & 927,180 & 363,468 & $8,155,756$ & $2,237,169$ \\
\hline Total number of admissions & $2,146,586$ & 786,678 & $18,779,688$ & $4,789,864$ \\
\hline Age, mean (SD) & $79.18(7.72)$ & $79.20(7.77)$ & $79.00(7.69)$ & $79.06(7.75)$ \\
\hline Male $(\%)$ & 41.87 & 42.08 & 41.91 & 42.21 \\
\hline \multicolumn{5}{|l|}{ Race $(\%)$} \\
\hline Black & 8.44 & 8.36 & 9.74 & 9.67 \\
\hline White & 87.90 & 87.88 & 85.37 & 85.21 \\
\hline Hispanic & 1.40 & 1.30 & 1.95 & 1.90 \\
\hline Other & 2.26 & 2.46 & 2.94 & 3.22 \\
\hline Elixhauser comorbidity score (SD) & $8.77(7.90)$ & $9.56(8.45)$ & $8.64(7.81)$ & $9.49(8.34)$ \\
\hline Dual eligible $(\%)$ & 21.16 & 19.04 & 23.54 & 22.53 \\
\hline \multicolumn{5}{|l|}{ Primary diagnosis \% (top 5) } \\
\hline $\begin{array}{l}\text { Major joint replacement of lower } \\
\text { extremity }\end{array}$ & 4.75 & 5.44 & 4.86 & 5.42 \\
\hline Heart failure and shock & 5.00 & 4.96 & 5.19 & 5.17 \\
\hline Simple pneumonia and pleurisy & 3.67 & 3.78 & 4.07 & 4.26 \\
\hline Septicemia or severe sepsis & 3.48 & 4.39 & 3.75 & 4.83 \\
\hline COPD & 3.49 & 3.23 & 3.80 & 3.71 \\
\hline \multicolumn{5}{|l|}{ Comorbidity \% (top 5) } \\
\hline Hypertension & 49.38 & 51.69 & 49.87 & 51.58 \\
\hline Hyperlipidemia & 38.51 & 46.01 & 37.37 & 44.12 \\
\hline Coronary atherosclerosis & 35.60 & 38.46 & 34.99 & 37.30 \\
\hline Cardiac arrthymia & 31.40 & 34.66 & 30.29 & 33.39 \\
\hline Fluid or electrolyte disorder & 28.38 & 31.43 & 29.21 & 32.3 \\
\hline Urban zipcode $(\%)$ & 94.45 & 94.12 & 91.62 & 91.68 \\
\hline \multicolumn{5}{|l|}{ Discharge location (\%) } \\
\hline SNF & 26.41 & 26.72 & 23.65 & 24.42 \\
\hline IRF & 3.93 & 4.14 & 3.64 & 3.81 \\
\hline Home with services (HHA) & 12.68 & 13.34 & 11.84 & 12.78 \\
\hline Home & 55.67 & 54.33 & 59.37 & 57.36 \\
\hline Other & 1.31 & 1.47 & 1.50 & 1.63 \\
\hline 30-day readmission rate & 16.76 & 15.60 & 16.44 & 15.75 \\
\hline 30-day mortality rate & 4.69 & 4.73 & 4.92 & 5.03 \\
\hline
\end{tabular}

ACO accountable care organization, SD standard deviation, SNF skilled nursing facility, IRF inpatient rehabilitation facility, HHA Home Health Agency, PAC post-acute care

changes in these characteristics between the early and late periods for ACO and non-ACO hospitals.

\section{Hospital and Market Characteristics}

A total of $13.7 \%$ of ACO hospitals had a SNF participating in the same ACO, $51.9 \%$ had a co-participating IRF, and over $25 \%$ had a co-participating HHA (Table 2). Markets with an ACO had more SNFs (73.4 versus 39.2), more IRFs (5.9 vs. 3.1), and more HHAs (51.7 versus 22.4 ) though differences in market concentration for PAC care were small. There were no significant differences in changes in the number of admissions at ACO hospitals versus non-ACO hospitals, while ACO markets experienced a small decline relative to nonACO markets $(-1480.1$ admissions, $P<0.01)$ (see Supplementary Tables 7 and 8). PAC discharge patterns did not change in markets with ACO hospitals versus nonACO markets (see Supplementary Table 9).

\section{Unadjusted Trends and Test of Parallel Trends}

Trends in PAC use were similar among the non-ACO hospitals and the ACO hospitals grouped by time of ACO-initiation (Fig. 1). The results of the test of parallel trends indicated that no consistent divergence before ACOs were implemented in the pre-2012 period, except for HHA, for which the magnitudes were very small (changes of 0.2 to $0.4 \%$ ) (see Supplementary Tables 10-13).

\section{Risk-Adjusted Differences in Post-Acute Care Utilization}

After risk adjustment, there were no significant changes in PAC utilization with ACO implementation at ACO hospitals compared to non-ACO hospitals (Table 3). In particular, there was no change in probability of discharge to PAC overall, with an estimated change of 0.000 percentage points $(P=0.89)$ or for discharge to each PAC type (change in SNF use 0.000 percentage points, $P=0.73$; change in IRF use $0.000, P=0.96$; change in HHA use $0.001, P=0.57$; and change in SNF/IRF combined $0.000, P=0.75$ ).

After stratifying the analyses by type of patient, we also observed no change in PAC use. This included the highest risk patients and those admitted with each of six common conditions (major joint replacement, hip fracture surgery, CABG, CHF, AMI, COPD) using PAC and when looking at PAC use overall and each type of PAC use individually. Further, when considering changes in patterns of PAC use conditional on discharge to PAC, we again do not observe any differences over time for $\mathrm{ACO}$ and non-ACO hospitals. 
Table 2 Characteristics of ACO and Non-ACO Hospitals and Their Markets in 2013*

\begin{tabular}{|c|c|c|}
\hline \multicolumn{3}{|l|}{ Hospital $(n=3336)$} \\
\hline & $\begin{array}{l}\text { ACO hospitals } \\
(N=233)\end{array}$ & $\begin{array}{l}\text { Non-ACO hospitals } \\
(N=3103)\end{array}$ \\
\hline $\begin{array}{l}\text { No. certified beds, mean } \\
\text { (SD) }\end{array}$ & $356.18(279.11)$ & $221.80(220.37)$ \\
\hline \multicolumn{3}{|l|}{ No. certified beds $(\%)$} \\
\hline$\leq 100$ & 10.30 & 34.97 \\
\hline $101-250$ & 31.76 & 33.64 \\
\hline$\geq 250$ & 57.94 & 31.39 \\
\hline \multicolumn{3}{|l|}{ Teaching status $(\%)$} \\
\hline Non-teaching & 65.67 & 81.31 \\
\hline Minor teaching (RB & 21.89 & 13.66 \\
\hline \multicolumn{3}{|l|}{ ratio $<0.25)$} \\
\hline Major teaching (RB & 12.45 & 4.99 \\
\hline ration $\geq 0.25$ ) & & \\
\hline \multicolumn{3}{|l|}{ Ownership (\%) } \\
\hline Government & 10.30 & 18.34 \\
\hline Not for profit & 82.40 & 58.06 \\
\hline For profit & 7.30 & 23.60 \\
\hline \multicolumn{3}{|c|}{ PAC in same hospital ACO (\%) } \\
\hline SNF & 13.73 & NA \\
\hline IRF & 51.93 & NA \\
\hline НHA & 25.32 & NA \\
\hline \multirow{2}{*}{$\begin{array}{l}\text { Urban ZIP code } \S_{\dagger}(\%) \\
\text { Market }(n=307)^{\dagger}\end{array}$} & 90.21 & 69.93 \\
\hline & $\begin{array}{l}\text { With } \operatorname{ACO}(N= \\
95)\end{array}$ & $\begin{array}{l}\text { Without ACO }(N= \\
212)\end{array}$ \\
\hline No. ACOs in market & $1.38(0.74)$ & $\mathrm{NA}$ \\
\hline \multicolumn{3}{|l|}{ No. ACOs in market (\%) } \\
\hline 0 & 0 & 100 \\
\hline 1 & 72.09 & 0 \\
\hline 2 & 20.93 & 0 \\
\hline $3+$ & 6.98 & 0 \\
\hline $\begin{array}{l}\text { ACO hospital admission } \\
\text { in market }(\%)\end{array}$ & $32.38(22.10)$ & NA \\
\hline \multicolumn{3}{|l|}{ No. PACs in market $(\%)$} \\
\hline SNF & $73.43(63.86)$ & $39.15(35.81)$ \\
\hline IRF & $5.91(6.01)$ & $3.09(3.36)$ \\
\hline HHA & $51.69(78.74)$ & $22.36(27.04)$ \\
\hline \multicolumn{3}{|c|}{ Herfindahl-Hirschman index, mean (SD) } \\
\hline Hospital & $\begin{array}{l}2171.96 \\
(1844.53)\end{array}$ & $3185.14(2022.89)$ \\
\hline SNF & $427.04(277.06)$ & $733.69(500.10)$ \\
\hline IRF & $\begin{array}{l}4248.73 \\
(2537.59)\end{array}$ & $5629.80(2703.61)$ \\
\hline HНA & $\begin{array}{l}1751.51 \\
(1104.40)\end{array}$ & 2033.96 (1365.04) \\
\hline Aggregate PAC & $392.31(275.00)$ & $622.50(423.50)$ \\
\hline
\end{tabular}

ACO accountable care organization, $R B$ ratio resident-to-bed ratio, $S D$ standard deviation, SNF skilled nursing facility, IRF inpatient rehabilitation facility, HHA Home Health Agency, PAC post-acute care

*ACO Hospitals defined as any hospitals in cohort that participated in MSSP through January 2013

${ }^{\top}$ Market defined by Dartmouth Atlas Hospital Referral Regions

${ }^{*}$ Herfindahl-Hirschman index is based on number of hospital or PAC admissions

${ }^{\xi}$ Hospital urban ZIP code designation was built using US Housing and Urban Development CBSA to ZIP code

\section{Differences in Medicare Payments and Length of PAC Use}

There were no significant changes in Medicare payments over time by PAC type for patients discharged from ACO versus nonACO hospitals. This was true for SNF $(-\$ 108.33$ per beneficiary, $P=0.08)$, IRF ( $\$ 119.46$ per beneficiary, $P=0.21)$, and HHA ( $\$ 1.65$ per beneficiary, $P=0.90)$ ) (Table 4). Length of PAC use did not significantly change for beneficiaries discharged from ACO hospitals compared to non-ACO hospitals over the study period at SNF ( 0.027 days; $P=0.92)$ or IRF ( -0.071 days; $P=$ $0.34)$ and HHA ( 0.018 visits; $P=0.86)$.

\section{Co-participation of Hospitals and PAC Providers in an ACO}

There were no changes in PAC use between $\mathrm{ACO}$ and nonACO hospitals over the study period when limiting the ACOhospital group to those that also had a PAC provider coparticipating in their ACO (Supplementary Tables 5 and 6).

\section{DISCUSSION}

Among all Medicare FFS beneficiaries admitted to hospitals that initiated participation in the first three waves of MSSP ACOs, we found no discernible changes in patterns of PAC utilization and Medicare spending compared to beneficiaries admitted to hospitals not participating in ACOs over this period. This lack of association was true among beneficiaries admitted for six conditions that most commonly use PAC as well as the most medically complex beneficiaries. Similarly, hospitals that participated in an ACO with a PAC provider did not change use of formal PAC relative to non-ACO hospitals. The lack of association between admission to an ACO hospital and use of formal or institutional post-acute care was striking - the effects we observe are statistical zeros that are robust to sub-groups and various definitions of utilization.

Our findings offer a contrast to prior evidence that PAC patterns for Medicare beneficiaries attributed to ACOs are associated with significant reductions in institutional PAC utilization and decreased SNF spending. ${ }^{4,}$, 12, 13 Our analysis differs in two important ways: first, we focus on the effect in ACO hospitals rather than among all attributed beneficiaries, regardless of whether those beneficiaries go to an ACO hospital; and second, we consider the spillover effect to all beneficiaries rather than just attributed beneficiaries. We sought to answer a different, but complementary question because only $16 \%$ of Medicare beneficiaries are attributed to an ACO and hospitals in ACOs may experience stronger incentives than non-ACO hospitals for hospital-wide care redesign: namely, do all beneficiaries admitted to an ACO hospital, regardless of whether they are eventually attributed to the ACO or not, experience changes in PAC use? ${ }^{7,8}$ Our findings suggest that ACO hospitals are not redesigning PAC referral patterns hospital-wide, but rather they are doing so for selected beneficiaries that would eventually be attributed to them. It seems that Medicare beneficiaries who are unlikely to be attributed to an ACO may not benefit from admission to an ACO's hospital. Possible reasons for this finding may include that MSSP provides only small financial incentives to ACO hospitals, directly proportional to the number of beneficiaries admitted from its participant ACO, or that system-wide changes take longer than our sample period. Further, there were trends toward fewer admissions at the market level during this time period, with larger reductions in markets with 

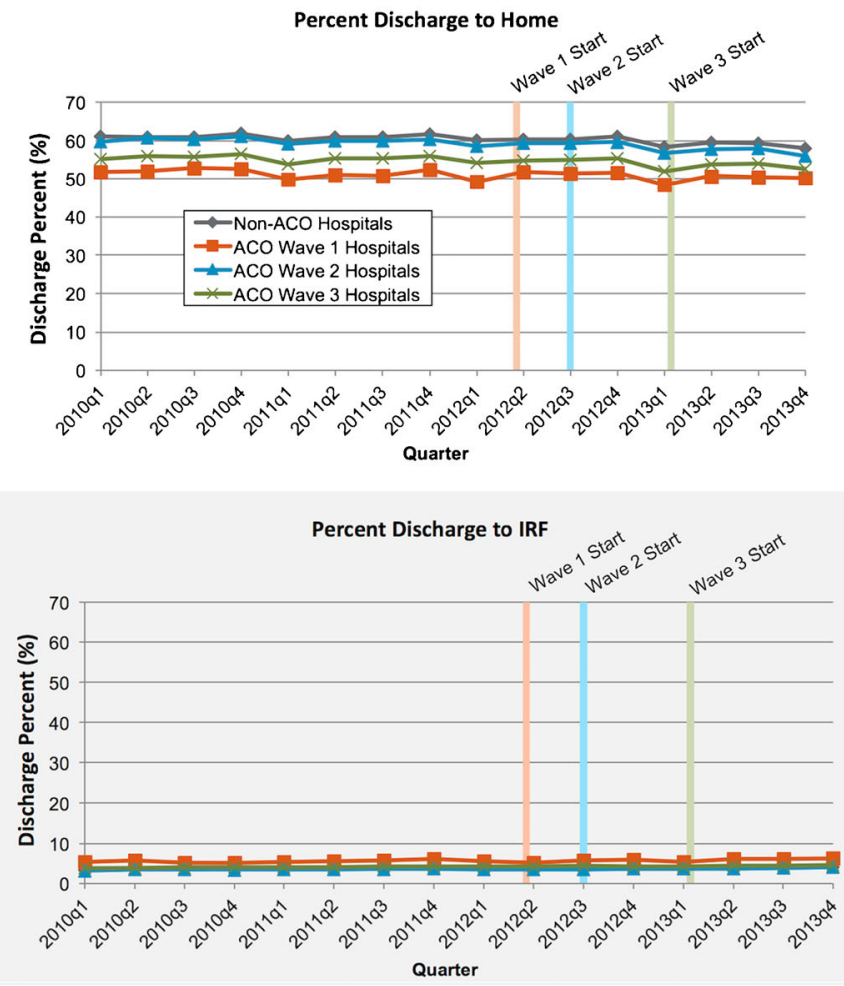

Percent Discharge to SNF Conditional on PAC Discharge

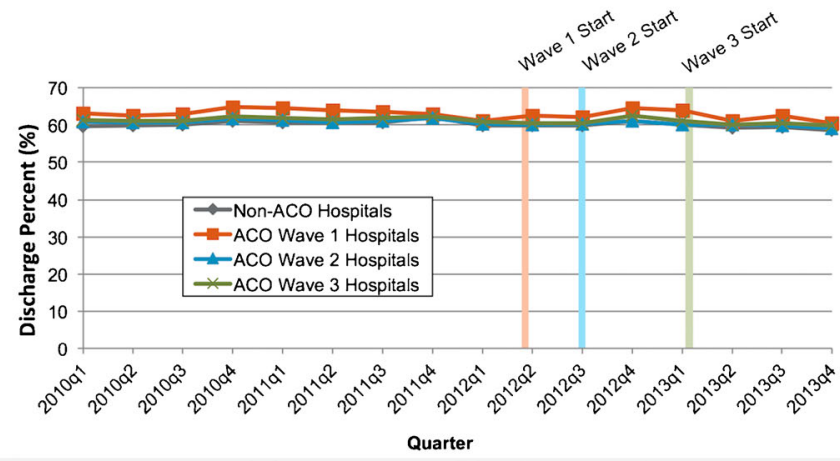

Percent Discharge to HHA Conditional on PAC Discharge

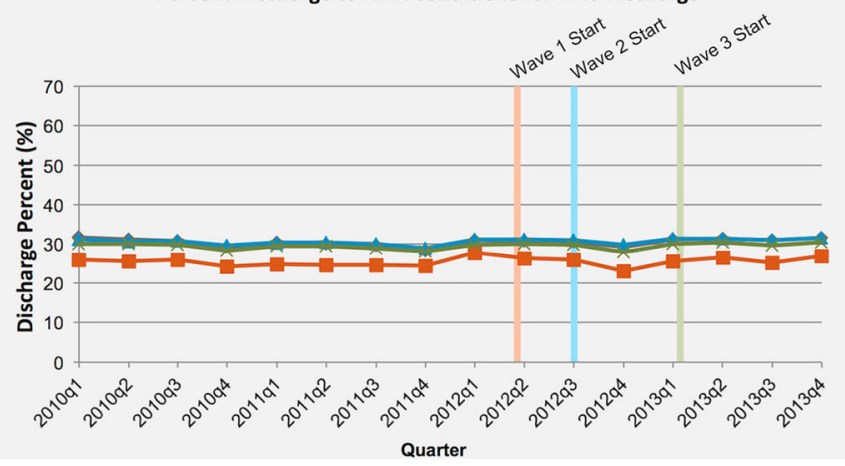

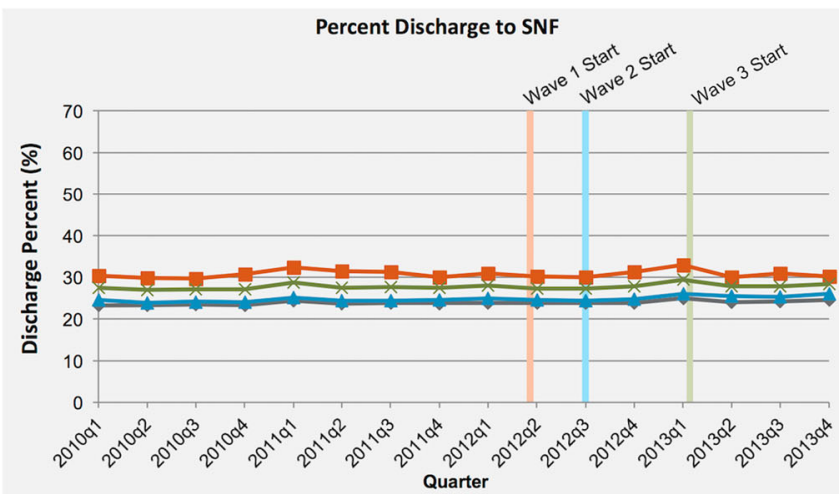

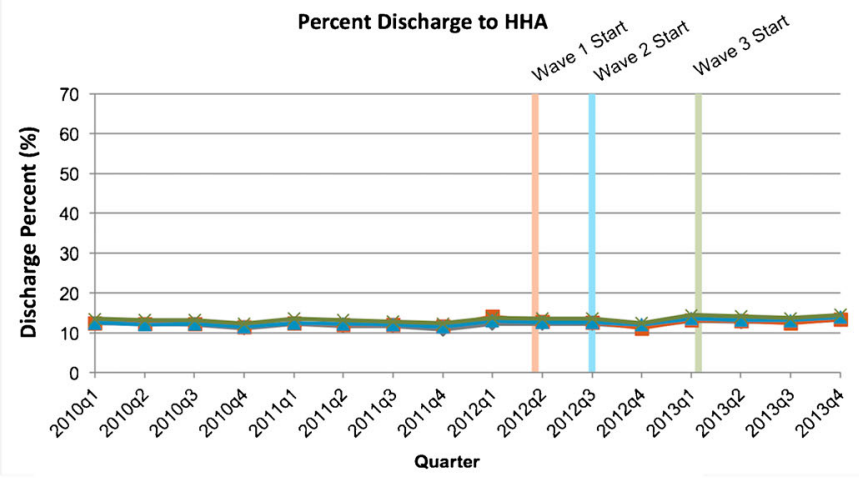

Percent Discharge to IRF Conditional on PAC Discharge

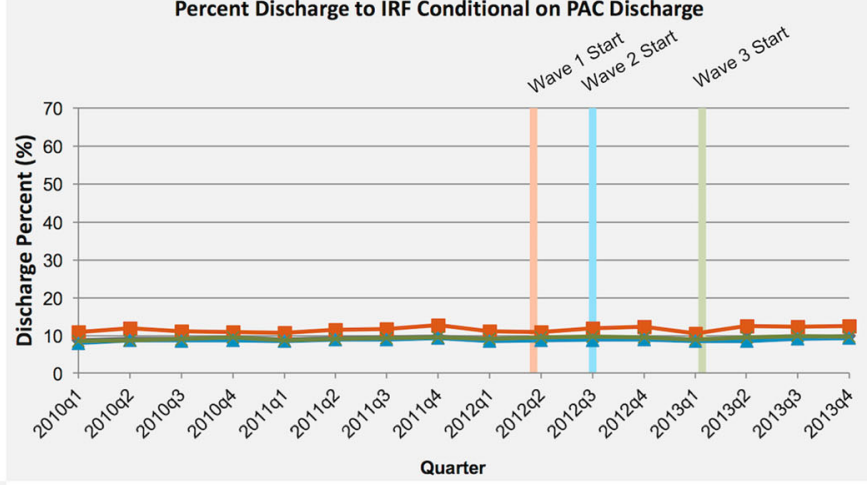

Fig. 1 Unadjusted proportion of discharges to each post-acute care (PAC) by ACO entry date and non-ACO status for each PAC type, 20102013. a Proportion of discharges to PAC type. Proportion of hospital discharges to particular post-acute care (PAC) provider types before and after initiation of ACO contracts. ACO waves 1 to 3 correspond to initiation of ACO contract on 04/01/2012, 07/01/2012, and 01/01/2013, respectively. The three colored vertical lines correspond to the start dates of the ACO waves with the same color. Overall, trends in discharges

to PAC were similar for ACO hospitals pre- and post-initiation of ACO contract and similar to that of non-ACOs in the study period. b

Proportion of discharges to PAC type conditional on PAC discharge. Proportion of hospital discharges to particular post-acute care (PAC) provider types among patients discharged to any PAC before and after initiation of ACO contracts. ACO waves 1 to 3 correspond to initiation of ACO contract on 04/01/2012, 07/01/2012, and 01/01/2013, respectively. The three colored vertical lines correspond to the start dates of the ACO waves with the same color. Overall, trends in discharges by PAC type conditional on discharge to PAC were similar for ACO hospitals pre- and post-initiation of ACO contract and similar to that of non-ACOs in the study period. 
Table 3 Differential Change in Probability of Discharge to Post-Acute Care from Admission to an ACO Hospital as Compared to Admission to a Non-ACO Hospital, by PAC type*

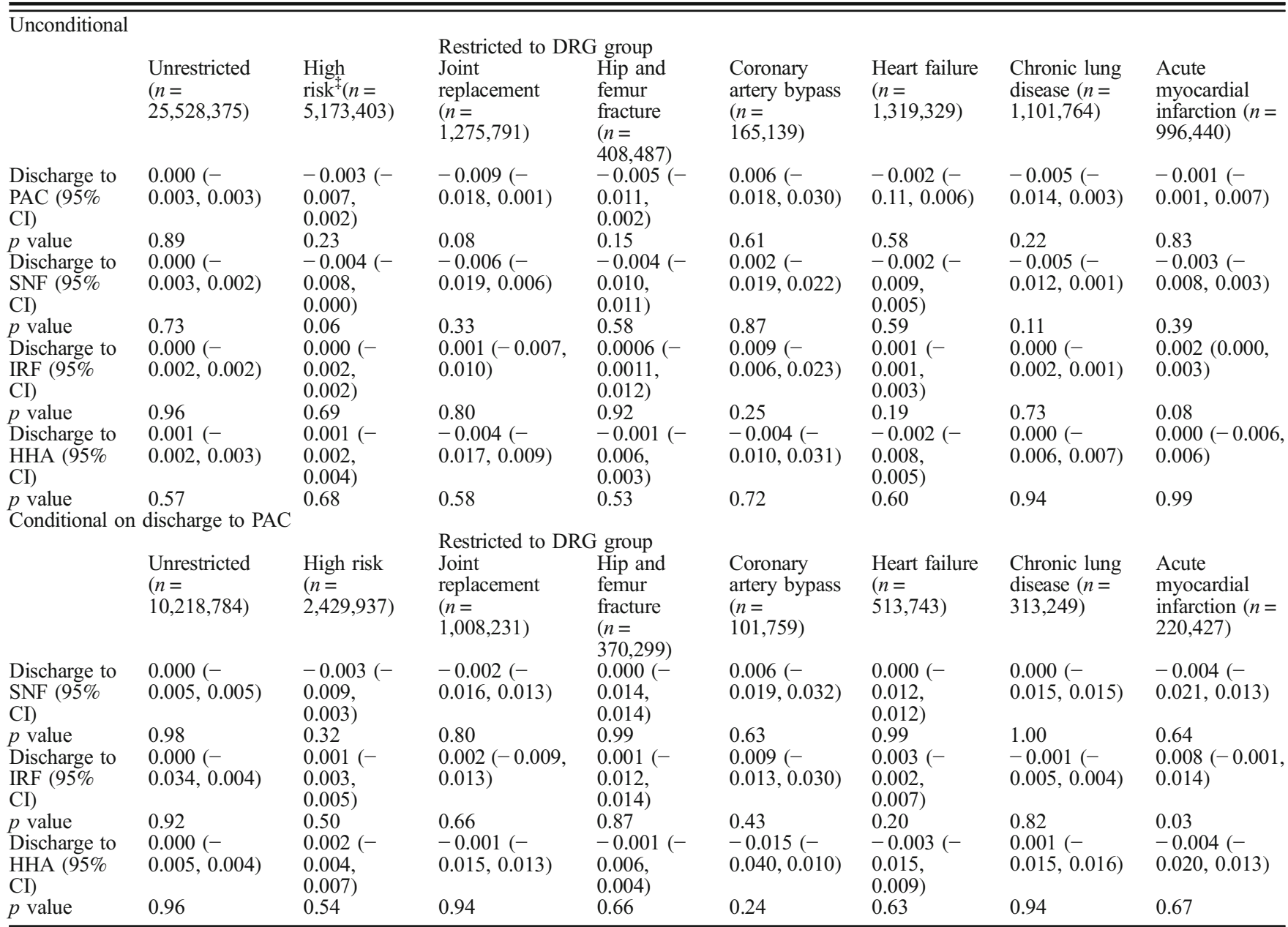

CI confidence interval, PAC post-acute care, SNF skilled nursing facility, IRF intermediate rehabilitation facility, HHA Home Health Agency

*Each coefficient represents a separate regression equation. Discharge location is the dependent variable. Model is a difference-in-differences linear probability model with hospital, hospital referral region, and time fixed effects. The base model estimates the change in probability of discharge to any PAC given admission to an ACO hospital versus a non-ACO hospital

${ }^{\prime}$ Beneficiaries are considered high risk if weighted Elixhauser Risk Index $\geq 16$ (top 20\% of sample)

ACOs, which may be correlated with unobservably sicker beneficiaries being admitted to hospitals and subsequently discharged to PAC.

Due to Medicare rules on attribution of beneficiaries, ACOs that do not include PAC services may not be incentivized to rein in spending on costly post-acute patients. ${ }^{14}$ However, our findings suggest that financial integration through co-participation of hospitals and ACOs may not be enough to offset the incentive to select beneficiaries for whom to change the PAC patterns. Further, the practice transformation needed to better target institutional PAC and reduce PAC spending does not seem to extend system-wide.

A final notable aspect is that conditions with traditionally high PAC utilization and geographic variation did not experience any changes, nor did beneficiaries with high illness severity. Of note, several of these conditions are included in

Table 4 Differential Change in Medicare PAC Payments and Length of Stay After Admission to an ACO Hospital as Compared to Admission to a Non-ACO Hospital, by PAC Type

\begin{tabular}{llll}
\hline \hline & SNF & IRF & HHA $^{*}$ \\
\hline Payment by PAC type (\$) (95\% CI) & $-108.33(-228.23,11.56)$ & $-119.46(-304.87,65.96)$ & $1.65(-24.22,27.53)$ \\
$p$ value & 0.08 & 0.21 & 0.90 \\
PAC length of stay (days) $(95 \%$ CI) & $0.027(-0.508,0.561)$ & $-0.071(-0.214,0.073)$ & $0.018(-0.214,0.073)$ \\
$p$ value & 0.92 & 0.34 & 0.86 \\
\hline
\end{tabular}

Difference in spending and PAC length of stay between ACO hospitals and non-ACO hospitals. Regression conditional on admission to corresponding PAC location type

CI confidence interval, PAC post-acute care, SNF skilled nursing facility, IRF inpatient rehabilitation facility, HHA Home Health Agency

* Length of stay for HHA corresponds to number of home visits 
Medicare's voluntary bundled payment program, which has shown large decreases in PAC use and spending for overlapping conditions like major joint replacement of the lower extremity $^{15,16}$ (also the subject of the mandatory Comprehensive Care for Joint Replacement (CJR) program). Our study suggests that a certain degree of caution should be placed on the expectations for ACO programs to curb high PAC use and variation nationwide, perhaps because incentives are not strong enough.

\section{Limitations}

Our study has a number of limitations. First, ACO hospitals may differ from non-participating hospitals in unobservable ways because the program is voluntary. However, by using hospital fixed effects, we compare each hospital to itself, thereby controlling for observed and unobserved characteristics of hospitals that do not change over time, though time-varying characterstics may still be confounders. Further, our analysis of "pre-" trends do not suggest systematic differences prior to ACO participation. Second, our analysis does not differentiate between effects on beneficiaries attributed to ACOs and those not attributed at the same hospital. However, our focus is on spillover effects and systemwide changes at ACO hospitals. Third, we focus on a sub-set of ACOs that include a hospital. While many ACOs are physicianled, it is critically important to understand how effective ACO policies are in influencing hospital practice patterns directly. Finally, we use administrative data, which includes limited clinical data on which to base risk-adjustment.

\section{CONCLUSIONS}

The admission of a Medicare beneficiary to a hospital participating in an MSSP ACO is not associated with hospital-wide changes in PAC utilization patterns, even when focusing on high-cost institutional PAC such as SNFs and IRFs. This precisely estimated zero effect is remarkably consistent for beneficiaries regardless of clinical condition, baseline level of comorbidity, and definition of utilization. Further, hospitals in ACOs that also include PAC providers similarly experience no changes relative to non-ACO hospital counterparts. Understanding the limitations of the impact of payment reforms such as ACOs can inform the rollout and future testing of policies that aim to improve health care value for all beneficiaries.

Acknowledgments: The authors would like to acknowledge Ning Chen for her contribution to this work. This research was funded by R01-HSO24266 by the Agency for Healthcare Research and Buality. Rachel Werner was supported in part by K24-AG047908 from the National Institute on Aging.

Corresponding Author: Amol S. Navathe, MD, PhD; Division of Health Policy, University of Pennsylvania, Philadelphia, PA, USA (e-mail:amol@wharton.upenn.edu).

\section{Compliance with Ethical Standards:}

The University of Pennsylvania Institutional Review Board approved the study.

Conflict of Interest: Dr. Navathe receives research funding from Hawaii Medical Services Association and Oscar Health Insurance. He also serves as an advisor to Navvis and Company, Navigant Inc., Lynx Medical, Indegene Inc., and Sutherland Global Services and receives an honorarium from Elsevier Press, none of which have relationship to this manuscript. All remaining authors declare that they do not have a conflict of interest.

\section{REFERENCES}

1. Medicare Payment Advisory Commission. March 2017 Report to the Congress: Medicare Payment Policy. Available at: http://www.medpac. gov/-documents-/reports. Accessed November 27, 2017.

2. Institute of Medicine (U.S.). Committee on Geographic Variation in Health Care Spending and Promotion of High-Value Care, Newhouse JP. Interim report of the Committee on Geographic Variation in Health Care Spending and Promotion of High-Value Care preliminary committee observations. Washington, D.C.: National Academies Press; 2013.

3. Chandra A, Dalton MA, Holmes J. Large increases in spending on postacute care in Medicare point to the potential for cost savings in these settings. Health Aff (Millwood) 2013;32(5):864-872.

4. McWilliams JM, Hatfield LA, Chernew ME, Landon BE, Schwartz AL. Early Performance of Accountable Care Organizations in Medicare. N Engl J Med 2016;374(24):2357-2366.

5. McWilliams JM, Chernew ME, Landon BE, Schwartz AL. Performance differences in year 1 of pioneer accountable care organizations. N Engl J Med 2015;372(20): 1927-1936.

6. McWilliams JM, Gilstrap LG, Stevenson DG, Chernew ME, Huskamp HA, Grabowski DC. Changes in Postacute Care in the Medicare Shared Savings Program. JAMA Intern Med. 2017;177(4):518-526.

7. Office of Enterprise Data \& Analytics CfMaMS. Medicare Enrollment Dashboard.Available at: https://www.cms.gov/Research-Statistics-Data-and-Systems/Statistics-Trends-and-Reports/Dashboard/MedicareEnrollment/EnrollmentDashboard.html. Accessed November 27, 2017.

8. Centers for Medicare and Medicaid Services. Fast Facts: All Medicare Shared Savings Program (Shared Savings Program) Accountable Care Organizations (ACOs). Available at: https://www.cms.gov/Medicare/ Medicare-Fee-for-Service-Payment/sharedsavingsprogram/Downloads/ All-Starts-MSSP-ACO.pdf. Accessed November 27, 2017.

9. Zaslavsky AM, Ayanian JZ, Zaborski LB. The validity of race and ethnicity in enrollment data for Medicare beneficiaries. Health Serv Res 2012;47(3 Pt 2):1300-1321.

10. Agency for Healthcare Research and Quality. Creation of New RaceEthnicity Codes and Socioeconomic Status (SES) Indicators for Medicare Beneficiaries: Final Report (January 2008). Available at: http://archive. ahrq.gov/research/findings/final-reports/medicareindicators/index. html. Accessed November 27, 2017.

11. Elixhauser A, Steiner C, Harris DR, Coffey RM. Comorbidity measures for use with administrative data. Med Care 1998;36(1):8-27.

12. Winblad U, Mor V, McHugh JP, Rahman M. ACO-Affiliated Hospitals Reduced Rehospitalizations From Skilled Nursing Facilities Faster Than Other Hospitals. Health Aff (Millwood) 2017;36(1):67-73.

13. Colla CH, Lewis VA, Bergquist SL, Shortell SM. Accountability across the Continuum: The Participation of Postacute Care Providers in Accountable Care Organizations. Health Serv Res 2016;51(4): 1595-1611.

14. McWilliams JM, Landon BE, Chernew ME. Changes in health care spending and quality for Medicare beneficiaries associated with a commercial ACO contract. JAMA 2013;310(8):829-836.

15. Dummit LA, Kahvecioglu D, Marrufo G, et al. Association Between Hospital Participation in a Medicare Bundled Payment Initiative and Payments and Quality Outcomes for Lower Extremity Joint Replacement Episodes. JAMA 2016;316(12): 1267-1278.

16. Navathe AS, Troxel AB, Liao JM, et al. Cost of Joint Replacement Using Bundled Payment Models. JAMA Intern Med 2017;177(2):214-222. 